\title{
The Role of Artificial Intelligence for Image Analysis in Surgical Pathology
}

\author{
Gabriela Izabela BALTATESCU ${ }^{\star}$, Mariana ASCHIE ${ }^{\star \star}$, Mariana DEACU ${ }^{\star \star \star}$, Lucian Cristian

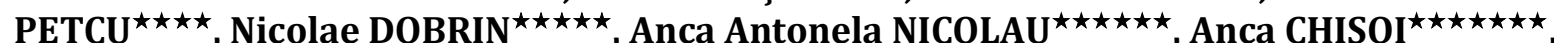 \\ Ionut BURLACU ${ }^{\star \star \star \star \star \star \star \star \star ~}$, Ionut Eduard IORDACHE ${ }^{\star \star \star \star \star \star \star \star \star \star ~}$, Liliana STERIU ${ }^{\star \star \star \star \star \star \star \star \star ~}$
}

\begin{tabular}{l} 
A R T I C L E I N F O \\
\hline Article history: \\
Accepted June 2020 \\
Available online August 2020 \\
\hline JEL Classification \\
C88, I10 \\
Keywords: \\
Artificial intelligence, Machine \\
learning, Whole slide images, \\
Surgical pathology
\end{tabular}

\begin{abstract}
A B S T R A C T
Nowadays, artificial intelligence (AI) is an important part of our life and it is a field which continues to grow, to develop and to be implemented in many aspects of our daily tasks. In addition, it continues to conquer many domains of health care with huge progress maid especially in medical image analysis, like radiography, computer tomography, magnetic resonance imaging, digital breast tomosynthesis, positron emission tomography scans or retinal images. The purpose of our work is to analyze the current state of AI systems and software in the practice of pathology. The digitalization process of pathology was possible due to development and improvement of several whole slide images systems in the last decade which provide a vast and rich image data to pathologists and researchers. The algorithmic base of AI is represented by machine learning (ML) and the basis of AI software reside in different statistical model and methods on large set of data. Initially, different mathematical models were used as toolset for ML like supervised and unsupervised learning, random forest, clustering algorithms or component analysis. In the present, deep learning subfield of ML with its neural networks (NN), artificial and convolutional NN, are frequently used in machine vision field. Other tools used in image analysis of digital slide are data augmentation, probability heat maps, patching and computer-aided diagnosis. All of them lead to a high valuable output from which an algorithm can be extracted and can assist the pathologist to render a final diagnosis. Even if there are a lot of challenges in integrated AI solutions in pathology department, they can streamline the diagnosis process in pathology with improvement of workflow, boosting the performance with a better and quicker diagnosis. Image analysis in pathology continues to expend and new branches emerge (digital pathology and pathology informatics) with huge potential for innovative
\end{abstract} discoveries.

(C) 2020 EAI. All rights reserved.

\section{Introduction}

Nowadays, technical systems endowed with artificial intelligence (AI) applications are playing an important part in our life. AI is a technology that can mimic human intelligence and represents a field which continues to grow, to develop and to be implemented in many aspects of our daily tasks. In addition, it continues to conquer many domains of health care with huge progress made especially in medical image analysis, like radiography, computer tomography, magnetic resonance imaging, digital breast tomosynthesis, positron emission tomography scans or retinal images. This outstanding extension of AI applications into the medical field has been possible due to increasing computing capacity combined with available huge database and applying new algorithms [1].

AI implies different advanced algorithms applied on high technologies with the specific task of delivering robust results from large and complex database in a similar way as human cognitive functions. One of the most complete definition of artificial intelligence (AI) is postulated by the European Commission's High-Level Expert Group on Artificial Intelligence in 2018: "AI refers to system designed by humans that, given a complex goal, act in the physical or digital world by perceiving their environment, interpreting the collected structured or unstructured data, reasoning on the knowledge derived from this data and deciding the best action(s) to take (according to pre-defined parameters) to achieve the given goal" [2].

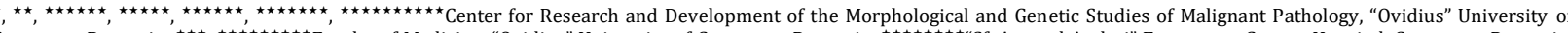

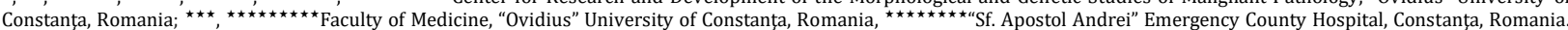
E-mail address: burlacuionut82@yahoo.com (Corresponding author) 
The main purpose of our work is to analyze the current state of AI systems in the practice of pathology medical field rendering an up-date of image analysis and tools of AI designed specifically for surgical pathology. The second one is to layout the most important information needed to create a cross disciplinary bridge between medical doctors, biostatistics, computer scientist and specialists from other disciplines that are willing to be involved in a multidisciplinary team aimed to expand and to implement the AI tools in pathology work-flow.

\section{Background}

In the surgical pathology medical field, image analysis (extraction of meaningful information from images) through AI help represent one of the most important application and it has been facing a tremendous growth, especially in the last ten years. The roots of computerized image analysis extend to 1965, when Mendelson et al (1965) first had approached and had studied the advantages and utility of cell morphology evaluation with computational help [3]. After this moment, the evolution of AI in the pathology have been developed with small steps untilwhole slide scanners devices appeared in 1990sand computational capacities have been improved. These machines have the capacity to scan usualtissue or cytological glass slides from surgical pathology department and digitalized images of them are obtained. The process of digitalization of the usual slides allowed the pathologist to have access to high quality images used for computer image analysis in education, diagnosis, numerous studies and clinical researches or in telepathology.

The role of surgical pathology in providing an accurate diagnosis and a correct classification of a disease to the clinician is a well-established fact. In addition, the histopathological reports deliver also important prognostic and predictive morphological, immunohistochemical and molecular features which will guide the clinicians to apply the proper and tailored therapy to the patient especially for different types of cancer or other chronic disease. Even if at the beginning of the pathology department the diagnosis was made only on the usual Hematoxylin-Eosin (HE) stain of the tissue slides, nowadays pathologists must reach to a conclusion after evaluating numerous slides from which they must extract a multitude of parameters with predictive and prognostic role (e.g. - grading and staging of a tumor, angiolymphatic invasion, number of atypical mitosis,tumor budding, the presence of tumor infiltrating lymphocytes - TILs, the presence of lymph node or distant metastases). In addition, doctors frequently must quantify different biomarkers on their immunohistochemical expression which have an important role for establishing an accurate diagnosis and for the initiation of a personalized therapy (e.g. estrogen and hormone receptors expression, overexpression for HER2neu protein, proliferate rate based on KI67 index). All of these tasks overload the amount of work for the pathologist and AI tools can help them to provide a complete, quick, accurate and objective morphopathological and immunohistochemical diagnosis reducing inter- and intra -pathologist variability.

AI, through its applications (machine learning and deep learning), has been involved in the diagnosis, research and teaching processes in pathology for the last two decades. More than that, due to new global health threats which haunts the well-being of mankind, the informatization of different domains increases its beneficial role and digitalization of pathology is also included in this process. It is an expansion domain with tremendous capacity to grow and we can already speak about a new branch of pathology field, like pathology informatics or computational pathology. For this reason, Salto-Tellez et al (2019) affirmed that integration of $\mathrm{AI}$ in pathology may represent "the third revolution in pathology" since the second one was considered to be implementation of molecular pathologyand the first revolution being immunohistochemical evaluation of biomarkers [4]. Awareness of the benefits of implementing AI lead to the launching of different public challenges on specific subjects and great results were obtained which further emphasized the importance of $\mathrm{AI}$ in pathology [5].

\section{Methods and results}

The current study used the most important web source for academic research, like PubMed [6] or Google Scholar [7].The following keywords were used for the research: „ digital pathology”, ,artificial inteligence”, „deep learning" and the period of time selected was between 2010-2020. The highligted data by these internet sources are overwhelming with 7273 on PubMed and 346000 on Google Scholar for „artificial intelligence” and „pathology”. „Digital pathology” is mentioned in 25700 journals and booksaccording to Google Scholar and 13300 by PubMed. Searching „artificial intelligence” and „digital pathology” on PubMed we noticed that the number of sources obtained is almost double between 2016 and 2020 (295 sources), comparing with 2010 - 2015 (155 sources). Searching for „deep learning” and „pathology” revealed 1224 
articles on PubMed and Google Scholar. The most important, recent and with high impact articles were selected and the most updated information which highlighted the most relevant data regarding artificial intelligence in pathology was extracted. The present study is both a descriptive research, presenting the benchmark data depicted in the references, and an observational and transversal type, interweaving the data regarding artificial intelligence, machine learning and digital pathology in the surgical pathology medical field

\section{Discussion}

For a long period of time, the workflow of a pathological laboratory remained unchanged even if some specific tasks of this process had been automated. Adoption of digital pathology is a slow and ongoing process and integrating AI tools in the pathology workflow is not an easy process all the time [8]. The digitalization process of pathology was possible due to development and improvement of several whole slide images (WSIs) systems in the last two decade which provide a vast, rich and high-resolution images to pathologists and researchers. Based on WSIs, the deployment image analysis techniques for analyzing and quantification of tissues slides was possible, which facilities and takes over some of the pathologist work [9].

One of the most useful utility of AI for surgical pathology resides in automated image analysis, but the implementation of AI was not a smooth process like in radiology due to the vast variables and difficulties present in a pathology image.Firstly, the scanned images are very large and must be broken in small tiles for processing them in both low or high magnification which finally must be integrated [10].Secondly, the variability of different patterns and tissues present in a WSI of usual tissue slide (different color, tissue and cell morphology which includes target tissue, stroma, angiolymphatic vessels, inflammatory cells, tumor cells and other complex phenotypes)require a trained pathologist for annotation of the regions of interest (ROI) [11]. Different computer vision techniques have been applied in order to detect ROI, like color space conversion, edge detection, image blurring, sharpening, pixel value quantization, clustering and thresholding [12]. Delimitation of ROI by a trained pathologist is very important as different morphological and immunohistochemical markers reside only in specific compartments of the surgical tissue [13]. Once a ROI is established than an algorithm is proposed and validated.

On the other hand, even if pathologists are highly trained to depict all the important features required to elaborate a diagnose and to evaluate the prognosis and the predictive markers, still they are prone to errors and some information from an image can't be easily extracted by the human visual system [11]. AI may intervene and come up with informatic algorithms in order to eliminate the biases and to help pathologists to fulfill their daily task $\mathrm{s}$ from the pathology laboratory and for this reason a lot of software tools provide nowadays numerous solutions [11].The base of $\mathrm{AI}$ in analyzing a WSIconsists in quantitatively mapping the image pixels into defined classes designated for different components of tissue structure [4].If, for a study, the images used are obtained by different image acquisition devices, it is strongly recommended that a normalization formula should be applied since there is some variability in the pixel sizes of the images [14]. The algorithmic base of $\mathrm{AI}$ is represented by machine learning (ML) and the basis of AI software consist in different statistical models and methods on large set of data. According to Ibrahim A. et al (2020) ML represents a "subfield of AI that develops algorithms that allows computer toadapt to a new problem without being reprogrammed", meaning that the computer is able to provide an output based on the available data through statistical methods [15]. ML compresses adaptive system that enable computers to learn from experience, by example or by analogy [16].

Initially, different mathematical models were used as toolset for ML which can be included in one of the three following categories (Figure 1): supervised learning which comprises classification and regression approaches [17]; unsupervised learning represented by clustering and dimensionality reduction algorithms [18]; reinforcement learning represented by policy algorithm [17]. The type of algorithm must be choose based on the type of the input data, which in medicine can be either images, text or numbers. In surgical pathology, images represent the most frequent type of input data and for this reason, visual recognition platforms were intensely developed. 


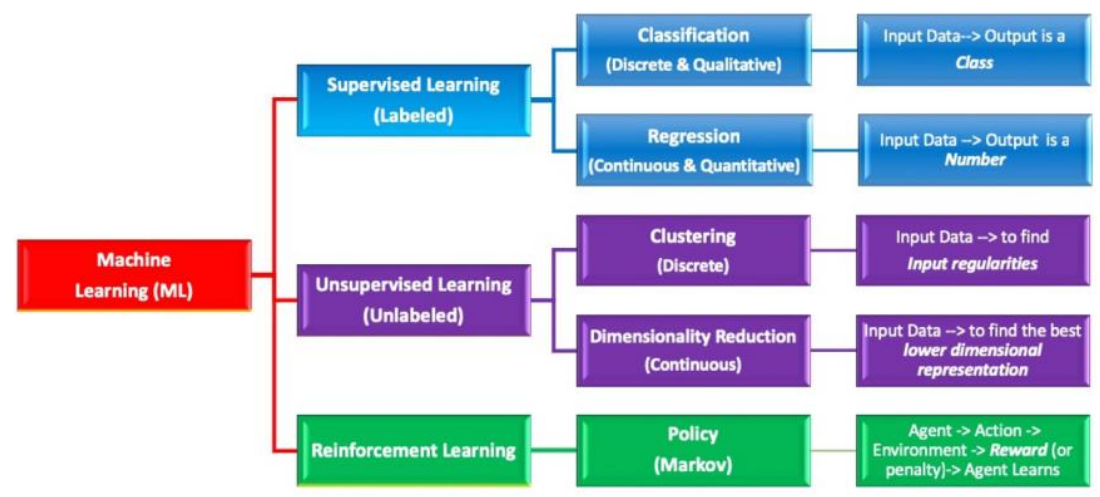

Figure 1. Diagram of machine learning algorithms according to Rashidi et al (2019) Source: Rashidi, H.H., Tran, N.K., Betts, E.V., Howell, L.P., Green, R. (2019) "Artificial Intelligence and Machine Learning in Pathology: The Present Landscape of Supervised Methods.” Academic Pathology, 6:2374289519873088.

Unsupervised deep learningis characterized by the ability to extract a meaningful histologically feature without a "training" or "labeled" data sets, being able to find patterns only from the input data distribution [19]. From this category of deep learning (DL), the most frequent algorithms included are hierarchical clustering and k-means [19].

Reinforcement learning has the main scope to facilitate the interaction between computational agents and the environment by learning through trial and error, by demonstration or both [20]. Zhu et al (2020) defined reinforcement learning as "a reward system for the computer program to maximize the rewards in order to search for the best solution" [19].

The most useful and successfully used algorithm model for automated image analysis is supervised ML algorithm in which a "training" image data set and an output also known as "labeled" data are used to predict future unknown pathological images [17].The presence of this "ground truth" is essential for supervised ML model in order to allow computers to develop algorithms without programmed instructions [11]. The most useful algorithms of the supervised DL used for classification and regression functions are: linear and logistic regression [21], gradient boosting [22], classification trees and random forest [19].The classifications models are depended to pixel-wise or sliding-window (patch central on a pixel of interest) approaches in order to fulfill objects detection, like cells, glands tubules, nuclei or images classification tasks [23;24]. Regression models have the role to detect and predict the position of objects, like cells or nuclei which is a challenging task as these morphological features are both extremely variable in shape and color and have the tendency to overlap making their separation difficult $[25 ; 10]$. To overcome this issue, the regression model was used by enforcing topological constraints (the pixels far away from object centers have lower probability values than those closer to centers) [10]. Another role of regression models is to provide a prognostic value for cancer, like H-scores in WSI for breast cancer [26] by enforcing topological/spatial constraints in deep neural network models [10].Another DL model used for supervised ML in digital pathology is segmentation based model which proved its efficiency in fully convolutional network based approaches [10]. This model is useful in solving instance segmentation problems in image analysis [10].The output of supervised ML can be discrete (qualitative) when the "classification" model is used for identification and classification of histopathology images, or continuous (quantitative), numerical output when "regression" model is used [17]. The main advantage of unsupervised ML model isthe ability to quickly identify unknown and unidentifiable patterns by humans in a scanned image or from a database.

In the present, deep learningneural network, a subfield of ML structured on a form of neural network,is frequently used in machine vision field. Neural networks (NNs) have the quality to be very flexible as they can rely on either supervised or unsupervised machine learning [19].A NN is thought to be analogue to the thinking model of the human brain [16] and it is composed by artificial neurons similar in structure as a biological neuron [19].

The morphological and functional unit of the brain is the neuron, a nerve-cell which can be designated as an information-processing unit (Figure 2a) [16]. These neurons are connected by synapses and the signals are transmitted from one neuron to another by releasing electro-chemical reactions. Ten billion neurons and 60 trillion synapses make the cognitive capability of the human brain to be difficult to replicate. An artificial NN tries to replicate the network of neurons of the brain and it is composed by different number of simple 
and highly interconnected processors also called neurons. The connection between them is made through weighted links (similar to a synapse): each neuron receive several inputs, like the biological dendrite of the neuron, and the output, which correspond to the axon, is single but it is splitting before reaching to other neurons, without fragmenting the signal (Figure 2b) [16]. In the artificial NNs the raw data represents the input data for one neuron or an output for other [16].

From the first AI application till present, there are several approaches and using a model similar with the human brain, in which the simpler structure is represented by a "perceptron" - a simple form of artificial neural network designed to recognize different patterns, is one of the most common [14]. Soft computing approach (fuzzy logic systems, evolutionary algorithms), based on artificial NN, is also frequently used when is enough to provide an approximate solution to problems [14]. Nowadays, the statistical learning approach is predominantly used in AI and for artificial NN linear regression [14]. In this situation, each artificial neuron has a weight value and is updated by gradient descent algorithm during backpropagation to minimize global loss function followed by nonlinearity to activate function [19; 27; 28]. As a result, it is possible to extract important features without the presence of "the ground truth" [19].

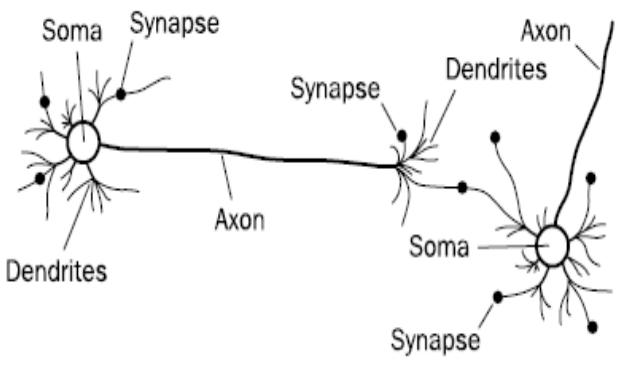

Figure 2a. Biological neural network Source: Negnevitsky, M. (2005) „Artificial intelligence: a guide to intelligent systems", $2^{\text {nd }}$ ed.

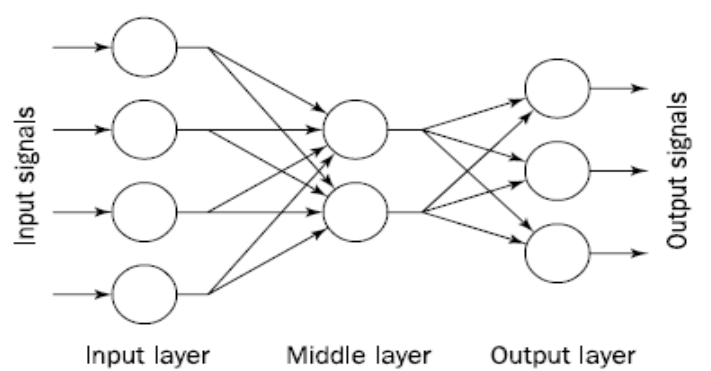

Figure 2b. Architecture of a typical artificial NN Source: Negnevitsky, M. (2005) „Artificial intelligence: a guide to intelligent systems", $2^{\text {nd }}$ ed

Using these algorithms allowed the AI to be empowered with features regarding identification of tissue patterns which were reserved to human visual cortex till now [4; 29] .DL is considered to be the next generation of ML, which is able to reproduce the human senses and is used not only in medical imaging but also for other tasks reserved only to human like face or voice recognition [30]. DL can be considered as a combination between traditional computer vision methods and modern ML approaches, in which the computer has the ability to select intermediate features and to apply a single model [11]. It can be differentiated from ML by the number of NNs which are from ten to hundreds compared with three or four layers encountered in ML. Another important feature which differentiate DL from ML refers to the way of learning: DL is able to give an output in a more independent way compared to ML which requires hard coded features to learn from [30]. All of these improvements allowed AI to move forward, completing tasks which were not possible in the past. Zarella et al. (2019) synthesized the application of DL as image segmentation, object classification, object recognition and clinical outcomes prediction [11]. Thus, at present, AI with these new tools manages to surpass the performances of the pathologist in certain aspects of this field of medicine, a fact that will be consolidated and expanded in the future [29].

Deep learning with its convolutionneural network (NN) algorithms are frequently used in the medical research. For structured data, fully connected NN are applied and for sequential data (text, audio), recurrent NN (RNN) are used; for image data convolutional NN (CNN) are considered [14; 19]. Nowadays, CNN are considered to be "the gold standard" for image classification and they are always taken into consideration when a lot of images are required to be evaluated, like in pathology medical field [17]. Jiang et al (2020) beautifully explained in its study the workflow of DL-based AI in pathology (Figure 3) in which the WSIs, obtained by scanning the usual tissue slides, are used to extract features from the patching images by different networks algorithms. All these features are then classified and, at the end of this machine hidden process, either a diagnosis or a prognostic model is deliver [31].The main advantage of them are the capacity to identify non-linear relationships but the risk of overfitting and the need for higher computational power and time are the main limitations [17]. 
Applications of DL architectures in pathology target some of the most common tasks performed by pathologist in the daily practice. It is used to differentiate a normal morphological tissue from an abnormal one, to establish the biological potential of different tumor cells proliferations (benign versus malignant), to establish the morpho-pathological type of the tumor and to grade it [32]. Another great feature ofDL algorithm is that it has the capacity to extract valuable information from the digitalized image that are not recognized by the human eyes and offer a new insight over the disease and discover unknown patterns from WSI (next generation morphology) [32;4].

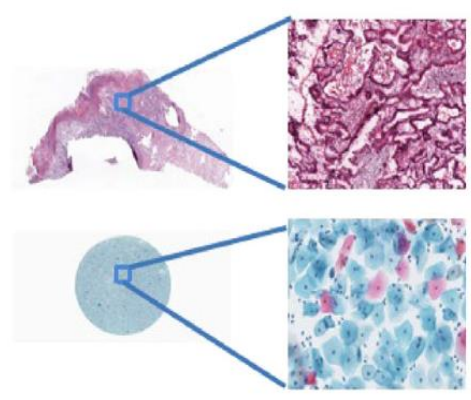

Whole-slide images Image patches Input

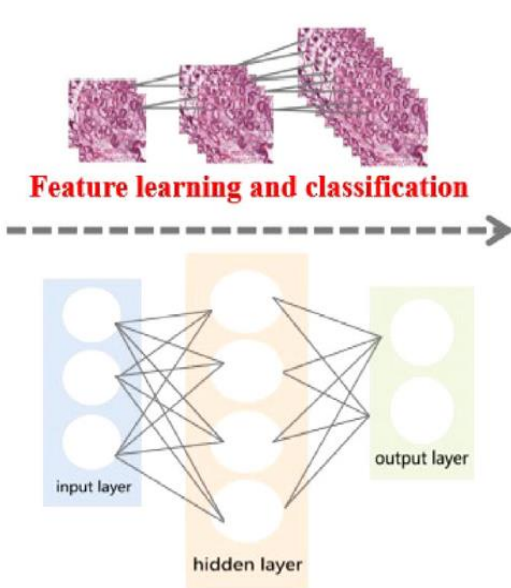

Enter into the neural networks

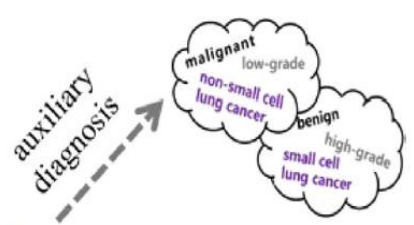

Output

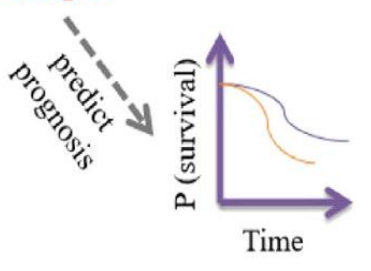

Different tasks

Different type of outputs

Figure 3. A schematic representation of the steps of DL-based AI in pathology according to Jiang et al (2020)

Source: Jiang, Y., Yang, M., Wang, S., Li, X., Sun, Y., (2020), "Emerging role of deep learning-based artificial intelligence in tumor pathology", Cancer Communications, 40, pp: 154-166.

If the first generation of $\mathrm{AI}$ was considered to be the traditional statistical analytics and the second one being attributed to DL with artificial NNs, the third generation of AI is considered to be represented by deep reinforcement learning which aimed to be similar to the human motor cortex and to be able to learn from errors. It can be considered that we are already in this phase as there are applications like Google DeepMind and GO, which fulfill the aforementioned criteria.

\section{Conclusion}

Even if there are a lot of challenges regarding integration ofartificial intelligence solutions in the pathology department, it can streamline the diagnosis process in pathology with improvement of workflow, boosting the performance with a better and quicker diagnosis.Almost all the tools of AI are ready to be applied for automated image analysis and to be integrated into digital pathology systems. Digital microscopy in conjunction with AI provides quantitative data, reproductible analysis and a high throughput which help the pathologist to overcome all the present tasks required in the daily work of the pathology laboratory. Image analysis in pathology continues to expand and new branches are emerging (digital pathology/pathology informatics) with huge potential for innovative discoveries.Deep learning is intensely applied in the medical field because is a very flexible model which accept different formats of data. Developing DL models suitable for WSIs analysis continues to represent a challenge task for digital pathology which is conquered day by day by due to pathologists and computer scientists dedicated to this field.

Pathologists and physicians must make the most of these new tools offered by computational development and the application of new algorithmic models. Artificial intelligence will make its presence felt more and more in the specialty of surgical pathology and it is important that everyone which come in contact with this new branch of medicine gets involved and stays informed with all the advances in technology and information. 


\section{Conflicts of interest}

The authors declare that they have no conflicts of interest

\section{Author contributions}

All the authors have the same contribution.

\section{Funding}

This work was supported by University "Ovidius" Constanta, Romania, through the grant number $5 / 14.11 .2018$

\section{Acknowledgments:}

This research was performed in the Center for Research and Development of the Morphological and Genetic Studies of Malignant Pathology from the "Ovidius" University of Constanța, POSCCE 2.2.1. Project ID: 1844, code SMIS:48750, CEDMOG, contract 627/11.03.2014.

This work is sustained by the project ANTREPRENORDOC, in the framework of Human Resources Development Operational Programme 2014-2020, financed from the European Social Fund under the contract number 36355/23.05.2019 HRD OP /380/6/13 - SMIS Code: 123847."

\section{References}

1. Vocke, C., Constantinescu, C., Popescu, D. (2019) "Application potentials of artificial intelligence for the design of innovation processes", Procedia CIRP, 84, pp. 810-813.

2. The European Commission's High-Level Expert Group on Artificial Intelligence Brussels, (2018). "A Definition of AI: Main Capabilities and Scientific Disciplines", Available on https://www.aepd.es/sites/default/files/2019-12/ai-definition.pdf; Accessed on December 2019

3. Mendelsohn, M.L., Kolman, W.A., Prewitt, J.M. (1965) “Computer analysis of cell images”. Postgraduate Medicine, 38, pp. 567-573.

4. Salto-Tellez, M., Maxwell, P., Hamilton, P. (2019) "Artificial intelligence-the third revolution in pathology", Histopathology, pp. 372-376.

5. Rotemberg, V., Halpern, A., Dusza, S., Codella, N.C. (2019) "The role of public challenges and data sets towards algorithm development, trust, and use in clinical practice", Seminars in Cutaneous Medicine and Surgery, 38(1), E38-E42.

6. https://pubmed.ncbi.nlm.nih.gov; Accessed on March 2020

7. https://scholar.google.com. Accessed on May 2020

8. Colling, R., Pitman, H., Oien, K., Rajpoot, N., Macklin, P., CM-Path AI in Histopathology. Working Group, Snead, D., Sackville, T., Verrill, C. (2019) "Artificial intelligence in digital pathology: a roadmap to routine use in clinical practice", Journal of Pathology, 249, pp. 143150.

9. Gurcan, M.N., Boucheron, L.E.,Can, A., Madabhushi, A., Rajpoot, N.M., Yener, B. (2009) "Histopathological image analysis: a review", Biomedical Engineering, IEEE Reviews, 2, pp. 147-171.

10. Srinidhi,_C.L., Cigab, O., Martela, A.N. (2019) "Deep neural network models for computational histopathology", Available on line in 28 Dec 2019on https://arxiv.org/abs/1912.12378.

11. Zarella, M.D., Bowman, D., Aeffner, F., Farahani, N., Xthona, A., Absar, S.F., Parwani, A., Bui, M., Hartman, D.J. (2019) “A Practical Guide to Whole Slide Imaging A White Paper From the Digital Pathology Association.”, Archives of pathology \& laboratory medicine, 143 (2), pp. 222-234.

12. Meijering, E. (2012) "Cell segmentation: 50 years down the road", [life sciences], IEEE Signal Processing Magazine, 29, pp. 140-145.

13. Aeffner, F., Wilson, K., Bolon, B., Kanaly, S., Mahrt, C.R., Rudmann, D., Charles, E., Young, G.D. (2016) “Commentary: roles for pathologists in a high-throughput image analysis team", Toxicologic Pathology, 44(6), pp. 825-834.

14. Chang, H.Y., Jung, C.K., Woo, J.I., Lee, S., Cho, J., Kim, S.W., Kwak, T.Y. (2019) "Artificial Intelligence in Pathology", Journal of Pathology and Translational Medicine, 53, pp. 1-12.

15. Ibrahim, A., Gamble, P., Jaroensri, R., Abdelsame, M.M., Mermel, C.H., Chen, P.H.C., Rakha, E.A. (2020) "Artificial intelligence in digital breast pathology: Techniques and Applications", The Breast, 49, e267 - e273.

16. Negnevitsky, M., "Artificial neural networks" (2005) in Negnevitsky, M (editor) "Artificial intelligence: a guide to intelligent systems", $2^{\text {nd }}$ edition, Addison Wesley, Pearson Education Limited, pp. 165 - 217.

17. Rashidi, H.H., Tran, N.K., Betts, E.V., Howell, L.P., Green, R. (2019) "Artificial Intelligence and Machine Learning in Pathology: The Present Landscape of Supervised Methods." Academic Pathology, 6:2374289519873088.

18. Buehler, L., Rashidi, H.H. (2005) "Bioinformatics Basics Application" in Biological Science and Medicine. 2nd ed. CRC Press, Taylor and Francis Group.

19. Zhu, W., Xie, L., Han, L., Guo, X. (2020) "The Application of Deep Learning in Cancer Prognosis Prediction", Cancers, 12,pp. 603.

20. Esteva, A., Robicquet, A., Ramsundar, B., Kuleshov, V., DePristo, M., Chou, K., Cui, C., Corrado, G., Thrun, S., Dean, J. (2019) "A guide to deep learning in healthcare", Nature Medicine, 25, pp. 24-29.

21. Cramer, J.S. (2003) "The Origins of Logistic Regression". SSRN Electronic Journal. Available on https://papers.tinbergen.nl/02119.pdf

22. Breiman, L., Friedman, J.H., Olshen, R.A. (2017) “Classification and Regression Trees”, $1^{\text {st }}$ edition, Routledge, New York, USA, Available on Taylor \& Francis eBooks.

23. He, K., Zhang, X., Ren, S., Sun, J. (2016) "Deep residual learning for image recognition", in: The IEEE Conference on Computer Vision and Pattern Recognition (CVPR), Las Vegas, NV, 2016, pp. 770-778.

24. Girshick, R. (2015) “Fast R-CNN" in: The IEEE International Conference on Computer Vision (ICCV). Santiago, 2015, pp. 1440-1448.

25. Sirinukunwattana, K., Ahmed-Raza, S., Tsang, Y.W., Snead, D.R., Cree, I.A., Rajpoot, N.M. (2016) "Locality sensitive deep learning for detection and classification of nuclei in routine colon cancer histology images", IEEE Transactions on Medical Imaging, 35, pp.11961206. 
26. Liu, J., Xu, B., Zheng, C., Gong, Y., Garibaldi, J., Soria, D., Green, A., Ellis, I.O., Zou, W., Qiu, G. (2019) “An end-to-end deep learning histochemical scoring system for breast cancer TMA", IEEE Transactions on Medical Imaging, 38, pp. 617-628.

27. Hinton, G.E. (1991) "Learning distributed representations of concepts", In Proceedings of the Eighth Annual Conference of the Cognitive Science Society, Hillsdale, NJ, USA, 7-10 August 1991; p. 12.

28. Bengio, Y. (2009), "Learning deep architectures for AI". Foundations and Trends in Machine Learning, 2, pp.1-127.

29. Litjens, G., Sanchez, C.I., Timofeeva, N., Hermsen, M., Nagtegaal, I., Kovacs, I., Hulsbergen-van de Kaa, C. Bult, P., van Ginneken, B., van der Laak, J. (2016), "Deep learning as a tool for increased accuracy and efficiency of histopathological diagnosis", Scientific Reports, 6:26286.

30. https://www.aiforia.com/deep-learning-vs-machine-learning/; Accessed on January 2020.

31. Jiang, Y., Yang, M., Wang, S., Li, X., Sun, Y., (2020), "Emerging role of deep learning-based artificial intelligence in tumor pathology", Cancer Communications, 40, pp: 154-166.

32. Acs, B., Rantalainen, M., Hartman, J. (2020), “Artificial intelligence as the next step towards precision pathology", Journal of Internal Medicine, published first on-line, https://doi.org/10.1111/joim.13030. 